\title{
A $p$-ADIC PROOF OF HODGE SYMMETRY FOR THREEFOLDS
}

\author{
KIRTI JOSHI
}

Dedicated to the memory of P $\bar{u}$. La. Deshpande.

\section{Introduction}

Let $X / \mathbb{C}$ be a smooth projective variety. Then it is a consequence of the Hodge decomposition theorem (see [6]) that for $p, q \geq 0$ we have:

$$
h^{p, q}=\operatorname{dim}_{\mathbb{C}} H^{q}\left(X, \Omega_{X / \mathbb{C}}^{p}\right)=h^{q, p}=\operatorname{dim}_{\mathbb{C}} H^{p}\left(X, \Omega_{X / \mathbb{C}}^{q}\right) .
$$

The Hodge theorem also asserts that these two spaces are complex conjugates and hence the equality of the two dimensions. In this note we give a $p$-adic proof of (11.1) when $X / \mathbb{C}$ is a smooth projective threefold. Our approach is based on the following observation: one first notes a purely $p$-adic assertion that (1.1) holds when Hodge numbers are replaced by more delicate $p$-adic invariants introduced by Ekedahl [3] called Hodge-Witt numbers. These invariants take in to account the torsion in the slope spectral sequence as well as the slopes of Frobenius in the crystalline cohomology of the variety. Hodge-Witt symmetry was proved by Ekedahl using his delicate duality theorem (we note here that we do not use Ekedahl's duality in the proof given below; we have replaced this by an elementary assertion $h_{W}^{0,2}=h_{W}^{2,0}$ ) as well as the crystalline Hard-Lefschetz theorem (when $X$ is a smooth projective threefold over a perfect field of characteristic $p$ ).

Once Hodge-Witt symmetry is proved one appeals to another result of Ekedahl (see [5]) which guarantees the equality of Hodge-Witt numbers and the Hodge numbers under suitable circumstances. That these required conditions are met when $X$ is a smooth projective variety over complex numbers is a simple consequence of Deligne-Illusie criterion (see [2]) for degeneration of Hodge-de Rham spectral sequence in characteristic $p$.

The restriction on dimension in Theorem 3.1 arises because Hodge-Witt symmetry is not known. Ekedahl gave a necessary and sufficient condition for Hodge-Witt symmetry to hold (in any dimension). This condition is given in terms of a certain equality of domino numbers for the slope spectral sequence. While this condition is not known to hold in dimension bigger than three, in Remark 3.2 and Scholium 3.3 we give one set of hypothesis under which these condition are satisfied. Further Ekedahl's conditions also hold, for instance, if $X$ is a smooth, projective HodgeWitt variety over a perfect field of characteristic $p>0$. Hence we deduce that if a smooth projective variety $X / \mathbb{C}$ has Hodge-Witt or ordinary reduction at an infinite set of primes then Hodge symmetry holds in characteristic zero.

We would like to thank Luc Illusie for correspondence and comments; we would also like to thank Minhyong Kim for discussions on Hodge symmetry and also to Jim Carlson whose lectures on mixed Hodge theory at the University of Arizona revived our interest in algebraic proofs of Hodge symmetry. We are grateful to

Date: Preliminary Version: 9/9/2000. 
F. Oort for his interest and his suggestions which have improved the readability of this paper. We also thank Dinesh Thakur for comments.

\section{Hodge-Witt Symmetry}

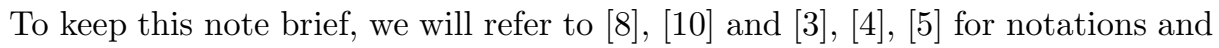
basic results. In this section $X / k$ is a smooth projective variety over a perfect field of characteristic $p>0$. Let $H^{j}\left(X, W \Omega_{X}^{i}\right)$ be the Hodge-Witt cohomology groups of $X$. Let $T^{i, j}$ be the dimension of the domino associated to the differential

$$
H^{j}\left(X, W \Omega_{X}^{i}\right) \rightarrow H^{j}\left(X, W \Omega_{X}^{i+1}\right)
$$

Let $m^{i, j}$ be the slope numbers associated to the slopes of Frobenius on the crystalline cohomology of $X$. For the definition see [9] or [5]. Then the Hodge-Witt numbers of $X$, denoted $h_{W}^{i, j}$ are defined to be

$$
h_{W}^{i, j}=m^{i, j}+T^{i, j}-2 T^{i-1, j+1}+T^{i-2, j+2} .
$$

Note that by [10] $T^{i, j}$ is zero if the corresponding differential of the slope spectral sequence is zero.

The following symmetry of slope numbers is a consequence of [1] and [14] and is due to Ekedahl (see [5]) we give his proof here for completeness.

Lemma 2.1. For any smooth projective variety $X / k$ over a finite field $k$ of characteristic $p$ and for all $i, j$ we have

$$
m^{i, j}=m^{j, i}
$$

Proof. Let for any rational number $\lambda h_{\text {cris }, \lambda}^{n}$ be the dimension (=multiplicity) of the slope $\lambda$ in $H_{\text {cris }}^{n}(X / W)$. Then by definition of $m^{i, j}$ we have

$$
m^{i, j}=\sum_{\lambda \in[i, i+1)}(i+1-\lambda) h_{\mathrm{cris}, \lambda}^{i+j}+\sum_{\lambda \in[i-1, i)}(\lambda-i+1) h_{\mathrm{cris}, \lambda}^{i+j}
$$

Thus to prove the equality of $m^{i, j}=m^{j, i}$ for all $i, j$ it suffices to prove that if $\lambda$ occurs in $H_{\text {cris }}^{n}(X / W)$ with some multiplicity, then $n-\lambda$ occurs in this cohomology with the same multiplicity. This is a consequence of Riemann hypothesis [14, and here is how one derives it from the Riemann Hypothesis. Let $\alpha_{1}, \ldots, \alpha_{r}$ be the eigen values of Frobenius on $H_{\text {cris }}^{n}(X / W)$. Then one knows by [1] and 14 that the characteristic polynomial of the Frobenius on crystalline cohomology is the same as the characteristic polynomial of Frobenius on $\ell$-adic étale cohomology for any $\ell \neq p$ (this is where we use the hypothesis that we are over a finite field. In particular this characteristic polynomial has integer coefficients and that the eigen values $\alpha_{i}$ are algebraic integers with $\alpha_{i} \overline{\alpha_{i}}=q^{n}$, where $q$ is cardinality of our finite ground field, and $q^{n} / \alpha_{i}$ is also an eigenvalue of Frobenius on this cohomology. Normalise the $p$-adic valuation on $W(k)\left[\alpha_{1}, \ldots, \alpha_{r}\right]$ so that $q$ has valuation 1 . Then it follows from this that if $\lambda$ is the slope of frobenius then $n-\lambda$ is also a slope of frobenius and it occurs with the same multiplicity.

We will also need the following elementary lemma due to Ekedahl (see [5]).

Lemma 2.2. Let $X / k$ be a smooth projective variety over a perfect field $k$ of characteristic $p>0$. Then

$$
h_{W}^{0,2}=h_{W}^{2,0}
$$


Proof. From the definition of $h_{W}^{0,2}$ and $h_{W}^{2,0}$ and Lemma 2.1 it suffices to prove that $T^{1,1}$ and $T^{2,0}$ are zero. The first is a consequence of 10 and the second is proved in 8 .

Remark 2.3. One can give a similar proof of the fact that $h_{W}^{0,1}=h_{W}^{1,0}=b_{1} / 2$, where $\frac{1}{2} b_{1}$ is the dimension of the Albanese variety of $X$ (see [5]).

Remark 2.4. Ekedahl has shown that $h_{W}^{i, j}=h_{W}^{j, i}$ for any smooth projective threefold $X / k$ and $i, j \geq 0$. The proof of the assertion $h_{W}^{i, 3-i}=h_{W}^{3-i, i}$ uses Ekedahl's duality theorem (see [3]). Ekedahl's duality is not sufficient to prove the corresponding assertion in higher dimensions.

\section{Hodge Symmetry for Threefolds}

Theorem 3.1. Let $X / \mathbb{C}$ be a smooth projective variety over complex numbers. Then we have $h^{i, j}=h^{j, i}$ for all $i+j \leq 2$. Moreover, if $X / \mathbb{C}$ is a smooth projective threefold then Hodge Symmetry holds for $X$.

Proof. Observe that $h^{i, 3-i}=h^{3-i, i}$ is a trivial consequence of Serre duality (see [7). Hence the assertion for threefolds follows from the first part of the assertion. So we have to prove $h^{i, j}=h^{j, i}$ when $i+j \leq 2$. Observe that $h^{0,1}=h^{1,0}$ follows by reduction to Picard variety of $X$, where the assertion is trivial. So we are left with proving that $h^{0,2}=h^{2,0}$.

We can assume, by a standard specialization argument, that $X$ is defined over a number field. We choose a proper, regular model of $X$ over a suitable open set of ring of integers of the number field. By further localisation on the base we may assume that model is smooth over the base and that the relative de Rham cohomology is torsion free and all the relative Hodge groups are torsion free as well. After further localisation we can assume that the Hodge numbers of every special fibre coincide with the Hodge numbers of the generic fibre and that the Hodge to de Rham spectral sequence of the special fibre degenerates at $E_{1}$. This can be done by the criterion of degeneration of the Hodge de Rham spectral sequence due to Deligne-Illusie (see [2]).

Let $X_{\mathfrak{p}}$ be a special fibre chosen as above. Then $H^{*}\left(X_{\mathfrak{p}}, W \Omega_{X_{\mathfrak{p}}}^{\bullet}\right)$ is a Mazur-Ogus object in the derived category of bounded complexes of modules over the CartierDieudonne-Raynaud algebra (see $[10]$ ) and hence we see that by $[5$. Corollary 3.3.1, page 86] one sees that $h_{W}^{i, j}=h^{i, j}$ and so by Lemma 2.2 we are done.

Remark 3.2. We note that of proof given above can be turned on its head: if $X$ is a smooth projective variety over complex numbers then as one does know that Hodge symmetry holds ([6]), so for sufficiently large primes, the reduction satisfies Hodge-Witt symmetry as the Hodge numbers and the Hodge-Witt numbers coincide by [2], [5]. In particular one deduces that Ekedahl's conditions on domino numbers (see 15, Proposition 3.2(ii), page 113]) are satisfied for almost all but finite number of reductions, regardless of whether or not these reductions are ordinary or HodgeWitt. So it would seems reasonable that at least under some reasonable hypothesis (liftablity to $W_{2}$, torsion-free crystalline cohomology), Hodge-Witt symmetry ought to hold in anay characteristic. We make this remark more precise in the following. 
Scholium 3.3. Let $X / k$ be a smooth projective variety over a perfect field of characteristic $p>0$. Assume that $X$ admits a smooth, projective lifting to $W(k)$ and $p>\operatorname{dim}(X)$ and the crystalline cohomology of $X$ is torsion free. Then for all $i, j$, the dimension, $T^{i, j}$ of the domino associated to the differential $d: H^{j}\left(X, W \Omega^{i}\right) \rightarrow$ $H^{j}\left(X, W \Omega^{j+1}\right)$ satisfies $T^{i, j}=T^{j-2, i+2}$.

Remark 3.4. In a forthcoming work (see 11]) we have investigated properties of Hodge-Witt numbers and slope numbers in detail. For instance we have shown that a weak version of the Bogomolov-Miyaoka-Yau inequality holds for all smooth projective surfaces of general type which lift to $W_{2}$ and have torsion free crystalline cohomology. We have also shown that the domino numbers of any smooth, projective variety which has torsion free crystalline cohomology are determined completely by their Hodge numbers and slope numbers. This was noted in [5] for Abelian varieties. Further we have also shown that for complete intersection in projective space, Ekedahl's conditions reduce to $T^{i, n-i}=T^{n-i-2, i+2}$ these are a consequnce of Ekedahl's duality for domino. These investigations will be reported in 11 .

Remark 3.5. Let $X / \mathbb{C}$ be a smooth projective variety. Then one expects that $X$ has Hodge-Witt or ordinary reduction modulo an infinite set of primes (see for instance 13 ). If one believes the truth of these assertions then by Ekedahl's work 5] and the above remarks, Hodge symmetry follows.

The method of proof is more general and can be used to prove the following:

Theorem 3.6. Let $X / k$ be a smooth projective threefold over a perfect field $k$ of characteristic $p>0$. Assume that the Hodge de Rham spectral sequence of $X$ degenerates and the crystalline cohomology of $X$ is torsion free. Then Hodge symmetry holds for Hodge numbers of $X / k$.

Remark 3.7. In general the conditions of Theorem 3.6 are not very easy to verify. But here is one application: assume that $X$ is Frobenius split and that $p \geq 5$. Then by a result of Mehta (see [12]) we see that Hodge de Rham spectral sequence of $X$ degenerates at $E_{1}$, so if crystalline cohomology of $X$ is torsion free then $X$ satisfies Hodge-Symmetry.

Remark 3.8. The assumption that the crystalline cohomology of $X$ is torsion free is a necessary assumption in Theorem 3.6. If this assumption is dropped then Hodge symmetry fails in positive characteristic. Here is one example (for surfaces): this is taken from [8, Chapter II, Section 7.3]. Let $X$ be a smooth projective Enriques surface in characteristic two. Assume that $X$ is singular, i.e., $H^{1}\left(X, \mathcal{O}_{X}\right)$ is one dimensional and frobenius is bijective on this vector space; such surfaces exist only in characteristic two. Then one has has a complete list of the Hodge invariants of $X$. In particular in the present situation, there are no global one forms on $X$ but as $H^{1}\left(X, O_{X}\right) \neq 0$, Picard scheme of $X$ is not reduced (it is equal to $\mu_{2}$ so the Albanese variety is zero and hence the second cristalline cohomology of $X$ has torsion (it is of type V-torsion in Illusie's classification of torsion). In fact as the proof of the 8 , Proposition 7.3.5, page 656] shows, the cohomology of $X$ with coefficients in the sheaf of Witt vectors is of finite type and so $\mathrm{X}$ is Hodge Witt. 


\section{REFERENCES}

[1] P. Deligne. La conjecture de Weil II. Publ. Math. I.H.E.S, 52:137-252, 1980.

[2] P. Deligne and L. Illusie. Relévements modulo $p^{2}$ et decomposition du complexe de de Rham. Invent. Math., 89(2):247-270, 1987.

[3] T. Ekedahl. On the multiplicative properties of the de Rham-Witt complex I. Ark. für Mat., 22:185-239, 1984.

[4] T. Ekedahl. On the multiplicative properties of the de Rham-Witt complex II. Ark. für Mat., $23,1985$.

[5] T. Ekedahl. Diagonal complexes and F-guage structures. Travaux ex Cours. Hermann, Paris, 1986.

[6] P. Griffiths and J. Harris. Principles of algebraic geometry. Wiley Classics Library. John Wiley and Sons, Inc., New York, reprint of the 1978 edition edition, 1994.

[7] R. Hartshorne. Algebraic Geometry. Number 52 in Graduate Texts in Mathematics. SpringerVerlag, New York-Heidelberg, 1977.

[8] L. Illusie. Complexe de de Rham-Witt et cohomologie cristalline. Ann. Scient. Ecole Norm. Sup., 12:501-661, 1979.

[9] L. Illusie. Algebraic Geometry Tokyo/Kyoto, volume 1016 of Lecture Notes in Mathematics, chapter Finiteness, duality and Künneth theorems in the cohomology of the de Rham-Witt complex, pages 20-72. Springer-Verlag, 1983.

[10] Luc Illusie and Michel Raynaud. Les suites spectrales associées au complexe de de Rham-Witt. Inst. Hautes Études Sci. Publ. Math., 57:73-212, 1983.

[11] Kirti Joshi. Crystalline aspects of geography of low dimensional varieties.

[12] Kirti Joshi. Exotic torsion in Frobenius split varieties. Preprint, 2000b.

[13] Kirti Joshi and C. S. Rajan. On frobenius splitting varieties and ordinary varieties. Preprint., 2000 .

[14] N. Katz and W. Messing. Some consequences of the Riemann hypothesis for varieties over finite fields. Invent. Math., 23:73-77, 1974.

School of Mathematics, Tata Institute of Fundamental Research, Homi Bhabha Road, Mumbai 400 005, india and Math. Department, University of Arizona, $617 \mathrm{~N}$ Santa Rita, Tucson 85721-0089, USA. 